\title{
The antioxidant effects of melatonin in blood platelets during exposure to electromagnetic radiation - an in vitro study
}

\author{
Małgorzata Lewicka1 ${ }^{1}$ Magdalena Zawadzka', Gabriela Henrykowska¹, Maciej Rutkowski², Andrzej Buczyński ${ }^{1}$
}

${ }^{1}$ Epidemiology and Public Health Department, Medical University of Łódź, Poland

${ }^{2}$ High School of Cosmetology and Health Science, Łódź, Poland

Abstract

Objectives: The article presents the results of an in vitro study aimed at identifying changes in parameters of oxidative stress - concentration of malondialdehyde (MDA), enzymatic activity of superoxide dismutase (SOD-1) and protective antioxidant role of melatonin (MLT) during the exposure of blood platelets to electromagnetic radiation (EMR) emitted by monitors.

Methods: Platelets were exposed to an EMR for 30 - and $60 \mathrm{~min}$. generated by monitors (1 kHz frequency, $220 \mathrm{~V} / \mathrm{m}$ intensity). In each sample the level of SOD-1 activity and concentration of MDA were determined.

Results: The MDA concentration increased significantly after 30 -and 60 -min. irradiation, as compared to control values (2.53 vs $1.36 ; 3.64 \mathrm{vs} 1.36 \mathrm{nmol} / 10^{\circ}$ blood platelets) and after the addition of MLT it decreased (2.53 vs $1.55 ; 3.64 \mathrm{vs} 1.12$ $\mathrm{nmol} / 10^{9}$ blood platelets). The activity of SOD-1 increased significantly compared to control values after $30 \mathrm{~min}$. and 60 min. of exposure to EMR (1.97vs $0.75 ; 2.08 \mathrm{vs} 0.75 \mathrm{U} / \mathrm{g}$ of protein), and significantly decreased after the addition of MLT only in samples exposed for $60 \mathrm{~min}$. (2.08 vs $0.95 \mathrm{U} / \mathrm{g}$ of protein).

Discussion: The results demonstrated the possibly negative effect of EMR on oxygen metabolism of blood platelets and indicated a possible protective role of melatonin in this process.

\section{INTRODUCTION}

Oxidative stress can be defined as a state of imbalance between the production and destruction of reactive oxygen species (ROS) arising from increased ROS generation and/ or reduced antioxidant enzyme activity. Such disturbances can cause toxic effects because high levels of ROS can cause cellular damage and apoptosis by oxidizing proteins, lipids, carbohydrates and nucleic acids. Oxidative stress can be caused by various environmental factors, including chemical and physical pollution, behavior factors, such as cigarette smoke and alcohol, as well as certain nutrients, such as saturated fat. In addition, a number of reports have examined the effects of electromagnetic radiation (EMR) exposure on the oxidative metabolism of cells [1, 2]; these range from increased lipid peroxidation caused by ROS to changes in the activity of antioxidant enzymes in various cells and tissues [3].

Melatonin (N-acetyl-5-methoxytryptamine) is a hormone with hydrophilic and lipophilic moieties that is produced in many tissues and organs and acts as an important enteric hormone in the gastrointestinal tract. However, it is synthesized mainly by the pineal gland according to the circadian rhythm. Its lipophilic properties enable it to penetrate all biological cell membranes, including the bloodbrain barrier. From a functional perspective, melatonin is involved in the regulation of the circadian rhythm of several 
biological functions and plays a key role in immunoregulation, reproduction, sleep, and inflammatory responses [4, 5].

Melatonin has also been shown to demonstrate cardioprotective effects in an experimental and a clinical studies. Sun et al. have reported that in cases of cardiac I/R injury, melatonin was in volved in both melatonin receptordependent and independent activity, the infarct size and cell death following cardiac I/R were reduced [6].

There are plenty of research which supports a protective role of melatonin in cancer treatment. Sonehara et al. have revealed that melatonin in concentration of $1 \mathrm{mM}$ has the inhibitory effect on the breast cancer cells line [7].

In addition to the above-mentioned qualities, numerous studies have reported that melatonin acts as an antioxidant due to its ability to be a potent free radical scavenger. It prevents oxidative stress by suppressing pro-oxidant enzymes, while stimulating the activity and expression of antioxidant enzymes [8].

Most significantly, melatonin also prevents oxidative damage in mitochondria, thus improving or preserving ATP (adenosine triphosphate) production, mitochondrial respiration, membrane potential and permeability transition. Consequently, it inhibits electron leakage and reactive oxygen species (ROS) production [9]. Anderson et al. have reported that melatonin has a positive effect on the development, immunization and intestinal health of infants by regulating the normal flora of the intestines [10]. A study by Zhu et al. was able to find that melatonin contributed to the increase in the number of Firmicutes in mice, suggesting a possible role for melatonin in preventing chronic inflammation in the bowel [11]. Due to melatonin's multiple actions as an anti-inflammatory, anti-oxidant, and anti-viral many authors suggest that it be used alone or in combination with currently-recommended drugs, to resist COVID-19 infection [4, 12].

However, the action of melatonin has been found to depend on concentration, incubation time and cell type. Wang et al. were surprised by their results, which showed that melatonin, a well-recognized ROS scavenging agent, dramatically enhanced ROS produced by the combination of Que and $\mathrm{Cu}$ (II) in a dose-dependent manner - the higher concentration of melatonin (from $10 \mu \mathrm{M}$ to $1000 \mu \mathrm{M}$ ) the more intense ROS production [13]. Other studies have found the activity of melatonin to be dependent on time [14]. Osseni et al. reported that melatonin exhibited antioxidant action in HepG2 cells after 24 hours incubation and became prooxidant after 96 hours [15].

Hence, it can be assumed that melatonin is also able to act as a pro-oxidant under certain circumstances and as an antioxidant under others. Therefore, further research is needed to estimate the potential side effects of long-term melatonin supplementation, as well as its concentration- and time-dependent effects.
Thus, the aim of this study was to investigate how melatonin influences selected parameters of oxidative stress, viz.malondialdehyde (MDA) and superoxide dismutase (SOD-1), during exposure to EMR under certain conditions.

\section{MATERIALS AND METHODS}

\section{Sample preparation}

Pig blood was collected from a slaughterhouse by exsanguination into $1 \%$ ethylene-diaminetetraacetic acid (EDTA). The obtained whole blood was fractionated by centrifugation at $1200 \mathrm{rpm} \times \mathrm{g}$ for $10 \mathrm{~min}$ at room temperature. Platelet rich plasma (PRP) was carefully drawn by pipette from the deposited layer of erythrocytes and transferred into polyethylene tubes. The PRP was then centrifuged again at $3000 \mathrm{rpm} \times \mathrm{g}$ for $15 \mathrm{~min}$. The precipitated platelets were suspended in $0.2 \mathrm{ml}$ of $0.9 \% \mathrm{NaCl}$. The obtained suspension of pig blood platelets was an input research model. Each test group consisted of 30 samples which were subjected to the next planned stages of the experiment. Each test group was divided into five series and made markings of the single stage for 6 samples. The operation was repeated to obtain adequate results for all 30 samples.

In this case, as the animals had been slaughtered for food, no approval was needed from the Ethics Commission.

\section{Incubation of platelets with melatonin}

Based on the composition of the samples, and existing data on melatonin dosage used in clinical trials, it was decided to use a mean dose of $15 \mathrm{mg}$ in the present study. The in vitro equivalent was $0.013 \mathrm{mM}$ : dissolved $3 \mu \mathrm{g}$ of solute (molecular weight $=232 \mathrm{~g} / \mathrm{mol}$ ) diluted with solvent to a total volume of exactly $1 \mathrm{ml}$. Briefly, $3.0 \mathrm{mg}$ of melatonin (cat. no M5250-1G, Sigma-Aldrich, USA) was weighed in a $10 \mathrm{ml}$ volumetric flask and ethanol was then added up to $10 \mathrm{ml}$, and this solution was taken for use in the study. Two $\mu$ l (containing $0.6 \mu \mathrm{g}$ of melatonin) of this solution was added to $0.2 \mathrm{ml}$ of a suspension of blood platelets, avoiding bright light. The sample was incubated in a dark place for 30 minutes before use in the study.

\section{Exposure condition setting and instruments}

To reconstruct the EMR generated by liquid-crystal display (LCD) monitors ( $1 \mathrm{kHz}$ frequency and $220 \mathrm{~V} / \mathrm{m}$ intensity), a flat capacitor acting as an electromagnetic field (EMF) source was positioned in a laboratory stand. Field measurements were performed according to the requirements of the Swedish Confederation of Professional Employees (Tjänstemännens Central Organization - TCO), which specifies strict conditions for the measurement of exposure [16]. 
The samples were exposed to EMR of $1 \mathrm{kHz}$ frequency and $220 \mathrm{~V} / \mathrm{m}$ intensity (corresponding to a distance of $15 \mathrm{~cm}$ from the monitor) for 30 and 60 minutes. The platelets were exposed on the day of collection from the slaughterhouse.

\section{Measurement of MDA - the marker of membrane lipid per- oxidation}

The study sample was prepared following method created by Placer [17], with 30 samples being used in each experimental group. The obtained results were expressed in $\mathrm{nmol} / 10^{9}$ of blood platelets.

The concentration of MDA was determined by measuring the absorbance at $532 \mathrm{~nm}$ using a T60 VIS (visible) spectrophotometer (OMC Envag company, Poland, Warsaw). Values were taken as relative to control samples $\left(1.8 \mathrm{~cm}^{3}\right.$ PBS (phosphate buffered saline) $+0.4 \mathrm{~cm}^{3}$ thiobarbituric acid).

\section{Measurement of the antioxidant activity of SOD-1 (Cu, $\mathrm{Zn}$ -} SOD) (EC. 1. 15. 1. 1)

The study samples (30 control and exposed samples) were obtained following method created by Misra and Fridovich [18]. The values were presented as $\mathrm{U} / \mathrm{g}$ of platelet protein. The amount of enzyme which causes a $50 \%$ inhibition at the maximal increase of absorbance by 0.025 of unit/min on a rectilinear segment of adrenochrome formation at $+25^{\circ} \mathrm{C}$ at $480 \mathrm{~nm}$ is defined as a unit of SOD-1 activity.

SOD-1 activity was measured at $480 \mathrm{~nm}$ using a T60 VIS spectrophotometer (OMC Envag, Warsaw, Poland) in both the control and study samples. Absorbance was measured at $+25^{\circ} \mathrm{C}$ every minute for $10 \mathrm{~min}$.

\section{Statistical analysis}

The arithmetic mean, standard deviation, median, minimum and maximum values were recorded. The groups were compared using the non-parametric Kruskal-Wallis ANOVA rank test and Mann-Whitney U-test. P-values $<0.05$ were considered as significant. Calculations were performed using Statistica software (license number 13.1.336.0).

\section{RESULTS}

Each sample, taken from an individual pig, was divided into six sub-samples. Each sub-sample was subjected to a different experimental treatment: 1. control, 2. control + melatonin, 3. radiation $30 \mathrm{~min}$., 4. radiation $30 \mathrm{~min}$. + melatonin, 5 . radiation $60 \mathrm{~min}$., 6 . radiation $60 \mathrm{~min}$. + melatonin. In each sample the level of MDA concentration and the level of SOD1 activity were determined.

\section{MDA concentration after the exposure to EMR}

The MDA concentration in blood platelets was significantly higher $(p<0.05)$ than control values after 30 minutes and 60 EMR exposure, as shown in table 1, figure 1.

\section{MDA concentration after the exposure to EMR and mela- tonin addition}

MDA concentration decreased in the sample treated with melatonin compared to the control sample; however, this decrease was not significant $(p>0.05)$. Among the blood samples exposed to EMR for 30 minutes, the samples treated with melatonin gave significantly lower MDA levels $(p<0.05)$ than those not treated with melatonin. After being exposed for 60 minutes, the samples treated with melatonin characterized with significantly lower MDA concentration than those without melatonin, as presented table 1 and figure 1.

\section{Enzymatic activity of SOD-1 after exposure to EMR}

Blood platelet SOD-1 activity was significantly higher due to radiation exposure than in the control samples $(p<0.05)$ after $30 \mathrm{~min}$. and after $60 \mathrm{~min}$. exposure: table 2 and figure 2.

\section{Enzymatic activity of SOD-1 after exposure to EMR + mela- tonin treatment}

Among the unexposed blood samples, significantly higher SOD-1 activity $(p<0.05)$ was observed in the sample treated with melatonin than in the untreated sample. For the samples exposed for 30 minutes, SOD-1 activity did not significantly differ in the sample with melatonin and the one without.

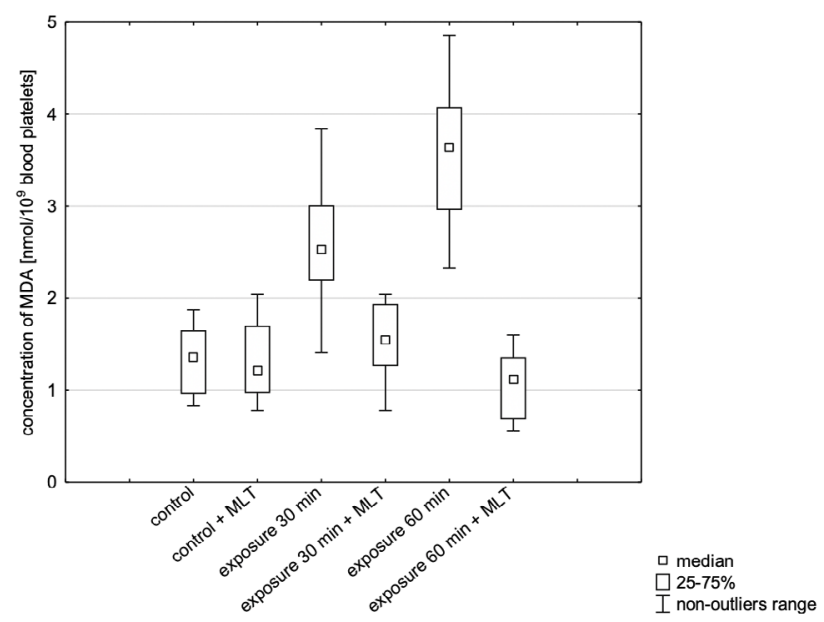

Figure 1. Changes of malondialdehyde (MDA) concentration in blood platelets exposed to the electromagnetic field with regard to changes in exposure time and application of melatonin (MLT) $(n=30)$. Variables were compared between groups using the nonparametric Kruskal-Wallis ANOVA and Mann-Whitney U-test. $P$-values below 0.05 were regarded as significant 
Table 1. The values (median \pm standard deviation) of MDA concentration in blood platelets treated with electromagnetic radiation dependent on exposure time and application of melatonin $(n=30)$

\begin{tabular}{ccccc}
\hline Individuals & Control & Control + melatonin & $\begin{array}{c}\text { Exposure } 30 \text { min. } \\
\text { 30 min.+ melatonin }\end{array}$ & $\begin{array}{c}\text { Exposure } \\
\text { melatonin }\end{array}$ \\
\hline MDA (nmol $/ 10^{9}$ blood platelets) & $1.36 \pm 0.36$ & $1.22 \pm 0.38$ & $2.53 \pm 0.64$ & $1.55 \pm 0.37$ \\
\hline
\end{tabular}

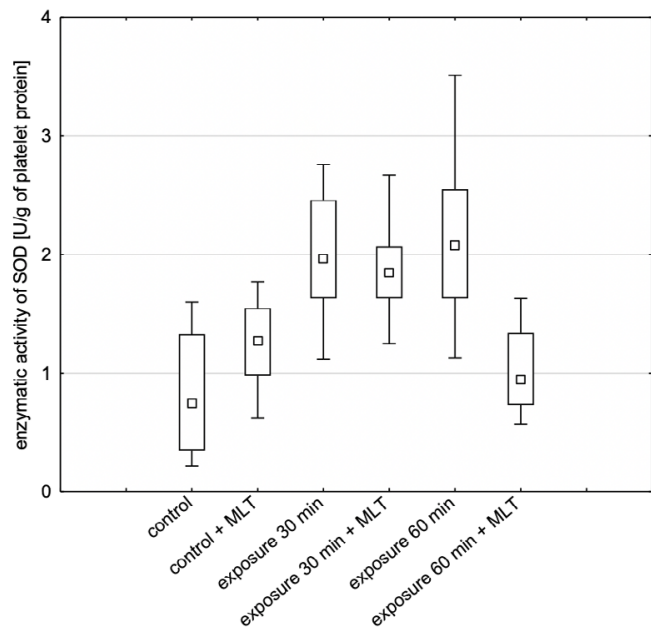

$\square$ median
$\square 25-75 \%$

non-outliers range

Figure 2. Enzymatic activity of superoxide dismutase (SOD-1) in blood platelets exposed to the electromagnetic field with regard to exposure time and application of melatonin $(M L T)(n=30)$. Variables were compared between groups using the nonparametric KruskalWallis ANOVA and Mann-Whitney U-test. P-values below 0.05 were regarded as significant

However, after 60 minutes of exposure, SOD-1 activity was significantly lower $(p<0.05)$ in the sample treated with melatonin, as presented in table 2 and figure 2 .

\section{DISCUSSION}

Several studies have examined the effects of exposure to EMR emitted by electric devices on oxidative stress. Oyewopo et al. reported that EMR of cell phone may induce oxidative stress, manifested as elevated MDA concentrations [19]. An in vivo study found that the exposure to static electric field with intensity of $56.3 \mathrm{kV} / \mathrm{m}$ for 7 and 14 days caused a temporary oxidative stress response, expressed only by the increase in activity of SOD and no significant increase was found in MDA content [3].
EMR with mean exposure of $4.09 \mathrm{~V} / \mathrm{m}$ and $16.27 \mu \mathrm{T}$ has also been found to be a potential oxidative stressor of musculoskeletal disorder, through its influence on the increased levels of MDA concentration and enzymatic activity of SOD and other enzymes, such as catalase and glutathione peroxidase in power plant workers [20].

In contrast, the presented study was conducted in vitro thus permitting a more focused analysis than in vivo (for example: precise quantification of exposure levels, provide the opportunity to eliminate external variables). Despite the abundance of literature available on the effects of EMR, little is still understood regarding its impact at the molecular level, as well as on the effects of various electric devices, such as computers and monitors, mobile phones, medical diagnostic and therapeutic equipment.

Presented study indicated that $30 \mathrm{~min}$. and 60 min. exposure to EMR emitted by LCD monitors was associated with elevated MDA concentration, a marker of lipid peroxidation. Such an increase may indicate attack by free radicals on the unsaturated fatty acids in the cell membrane, which would imply insufficient adaptation of the blood platelet antioxidant capacity, protecting against oxidation of cell membrane components. As a result, the destruction of membrane lipids and the end-products of this process, such as MDA, damages cells. This is a crucial step in the pathogenesis of several disease states such as atherosclerosis, IBD (irritable bowel syndrome), ROP (retinopathy of prematurity), BPD (borderline personality disorder), asthma, Parkinson's disease, kidney damage and preeclampsia.

The study also examined the effect of radiation on SOD-1 activity. It was found that exposure to EMR emitted by LCD monitors altered the activity of SOD-1 in blood platelets, with the enzyme activity increasing relative to control samples after 30- and 60-minute irradiation by a $220 \mathrm{~V} / \mathrm{m}$ intensity field. These changes in SOD-1 enzyme activity are probably

Table 2. The values (median \pm standard deviation) of enzymatic activity of SOD-1 in blood platelets treated with electromagnetic radiation dependent on exposure time and application of melatonin $(n=30)$

\begin{tabular}{|c|c|c|c|c|c|c|}
\hline Individuals & Control & $\begin{array}{l}\text { Control + mela- } \\
\text { tonin }\end{array}$ & Exposure $30 \mathrm{~min}$. & $\begin{array}{c}\text { Exposure } \\
\text { 30min.+ melatonin }\end{array}$ & Exposure $60 \mathrm{~min}$. & $\begin{array}{l}\text { Exposure } 60 \mathrm{~min} . \\
+ \text { melatonin }\end{array}$ \\
\hline SOD (U/g protein) & $0.75 \pm 0.48$ & $1.27 \pm 0.34$ & $1.97 \pm 0.51$ & $1.85 \pm 0.39$ & $2.08 \pm 0.64$ & $0.95 \pm 0.33$ \\
\hline
\end{tabular}


related to the higher concentration of free radical substrates induced by exposure to EMR.

In the samples where melatonin was added, SOD-1 was also altered. Greater enzyme activity was observed after adding melatonin (in unexposed controls). However, SOD1 activity in the EMR exposed samples was decreased by adding melatonin, with a significant reduction observed after 60 minutes of exposure.

It is possible that melatonin may act as an antioxidant by scavenging existing free radicals; this may have decreased SOD-1 activity by reducing the amount of free radical substrates. However, it is also possible that the incubation time of melatonin (30 min.) was too short to activate Nrf2 (Nuclear factor erythroid 2-related factor 2), which plays an important role in the activation of antioxidant enzymes.

Melatonin treatment also influenced the MDA content of blood samples, with MDA decreasing after the addition of melatonin in unexposed controls (i.e. control vs control + melatonin). In the blood samples exposed to EMR for 30 $\mathrm{min}$. and $60 \mathrm{~min}$., the MDA concentration was significantly lowered by the addition of melatonin, especially after $60 \mathrm{~min}$. of exposure.

The reduction in MDA content may be accounted for by the fact that although melatonin acts as an antioxidant in the presented study, it would also reduce the levels of free radicals. Such a reduction would inhibit the peroxidation of cell membranes, and thus reduce the amounts of MDA: this being the final product of the process.

Some studies also have reported that melatonin reduces lipid peroxidation. Bicer et al. noted that the cell damage caused by acute swimming exercise and diabetes in the bone tissue of rats could be prevented by melatonin supplementation [21]. In a study of irradiated animals, reduced MDA levels were found in the testicular tissue of those treated with melatonin [22]

In addition, melatonin has also been found to modulate enzyme activity of SOD [23].

The oxidant-antioxidant balance is maintained enzymatically, inter alia by superoxide dismutase (SOD), catalase (CAT), glutathione peroxidase (GPx) and nonenzymatically, by low molecular-weight substances such as melatonin and vitamins A (tretinoin), C (ascorbic acid) and $E$ (tocopherol). As the low molecular weight antioxidants are less specific than the antioxidant enzymes, they tend to act more as universal protectors which perform several functions and serve as the second line of defense, degrading the ROS which are not removed by SOD and CAT. Thus, antioxidant supplements may be effective in lowering oxidative stress that cannot be controlled by endogenous antioxidants.

However, there are claims that the current RDA (recommended daily allowance) levels of vitamin $C$ and $\mathrm{E}$ are too low to prevent oxidative stress, and it is possible that ingesting high amounts of supplements with antioxidant potential may lead to prooxidant effects or to "antioxidative stress" [24]; in fact, large doses of beta-carotene were found to increase the rate of lung cancer in a high-risk group of smokers [25].

Recent research years have suggested that melatonin also exerts pro-oxidant effects under certain circumstances and the action of this substance depends on concentration, incubation time and cell type. Kocyigit et al. have demonstrated that melatonin in low doses $(0.031-0.06 \mathrm{mM})$ increased cell proliferation and decreased ROS generation, but in higher doses $(0.125-5 \mathrm{mM})$ markedly inhibited the cell viability, induced DNA damage, apoptosis and ROS generation. Cytotoxic, genotoxic, apoptotic and ROS generating effects were significantly higher in cancer cells than those observed in normal cells [26]. Bejarano et al. have shown a pro-oxidant action of melatonin by inducing a rise of intracellular ROS in tumour cell lines [27]. Melatonin has been found to increase ROS and induce apoptosis in human platelets when administered at a concentration of $50 \mu \mathrm{M}-1$ $\mathrm{mM}$. This may indicate that the effects of high melatonin concentrations are also dependent on cell type, i.e. that it can influence cancer cells in a different way to normal cells [23].

Some studies also suggest that pro-oxidant and antioxidant activity may be influenced by the duration of treatment. Melatonin administered in concentrations of 0.1$10 \mu \mathrm{M}$ exhibited antioxidant activity in HepG2 cells after 24 hours incubation, but became pro-oxidant after 96 hours [15].

It is important to note that the above in vitro studies on the pro-oxidant properties of melatonin used pharmacological concentrations; however, various dosages can be used in therapy. Melatonin can be used as a hormone and/or chronobiotic for treating disturbances of the sleep-wake rhythm (e.g. due to shift work) and disorders of pathological sleep caused by changes of time zones. In these cases, the doses recommended by the manufacturers [28] of melatonin preparations and scientific studies range from $0.1-10 \mathrm{mg} /$ day with the exact dose being adjusted to individual patients. Producers usually recommend a dosage of $1-5 \mathrm{mg} /$ day for the first use and 2-3 mg/day for the second application.

The second type of use is an innovative application, i.e. using melatonin as a strong antioxidant or/and immunostimulator. In this case, it is often given as an adjunct to essential therapy for a range of civilization diseases and disorders caused by various chemical and physical factors. As the producers do not mention such applications, they typically do not provide any recommended doses: such data is restricted to scientific studies based on clinical trials [29]. Such doses are much higher than those used for traditional applications. For example: cluster headaches $10 \mathrm{mg}$ in the evening, sarcoidosis $20 \mathrm{mg} / \mathrm{day}$, systemic sclerosis $8-16 \mathrm{mg} /$ 
day, neurodegenerative diseases 8-24 mg/day, fatty liver disease $10 \mathrm{mg} /$ day, tumors (with chemo- or radiotherapy) 10 $50 \mathrm{mg} /$ day and cataracts (premedication before surgery) 10 $\mathrm{mg} /$ day. Interestingly, a prophylactic dose of $20 \mathrm{mg}$ before the threat is recommended for protection against ionizing radiation.

The mean dose chosen for the present study $(15 \mathrm{mg})$ was chosen based on these previous uses for innovative applications.

The consensus within the literature is that melatonin is a strong antioxidant; however, its anticancer, oncotherapeutic, antitoxic, immunostimulating, radioprotective, and antioxidative possibilities remain poorly understood, and little is known about its side effects. Further studies of melatonin are merited, especially with regard to its potential to protect against free-radical-related diseases.

\section{CONCLUSIONS}

1. Exposure to EMR emitted by LCD monitors ( $1 \mathrm{kHz}$ frequency and $220 \mathrm{~V} / \mathrm{m}$ intensity) influences the concentration of MDA, a marker of lipid peroxidation in pig blood platelets in vitro. After 30 and 60 min. irradiation, the MDA concentration increases relative to control values.

2. Thirty and $60 \mathrm{~min}$. EMR irradiation from LCD monitors increases SOD-1 activity in pig blood platelets relative to controls.

3. Exposure to examined EMR may cause adverse effects within blood platelet oxygen metabolism, potentially resulting in physiological dysfunction; however, this is an in vitro study and the results cannot be directly extrapolated to in vivo conditions.

4. The changes in SOD-1 and MDA levels observed in the blood samples after the addition of melatonin may indicate that melatonin has antioxidant properties.

\section{Acknowledgements}

This paper is dedicated to the memory of our co-worker, Professor Krzysztof Pacholski, who recently passed away. It was a great honor to work with him.

\section{Authors' Contribution}

M.L.: carrying out the experiments, writing the manuscript, literature review, analysis and interpretation of data, final proofreading and approval of the version for publication; M.Z.: designing the figures, analysis and interpretation of data; G.H.: carrying out the experiments; M.R.: research concept and design; A.B.: supervising the project, drafting the article or revising it critically for important intellectual content, research concept and design.

\section{Conflict of Interest}

The authors have no potential conflicts of interest to declare.

\section{Ethics Approval}

Due to the nature of the research, the consent of the ethics committee was not required.

\section{References}

[1] Lewicka M., Henrykowska G.A., Pacholski K., Szczęsny A., Dziedziczak-Buczyńska M., Buczyński A.: The impact of electromagnetic radiation of different parameters on platelet oxygen metabolism - in vitro studies. Adv. Clin. Exp. Med., 2015; 24: 31-35

[2] Yokus B., Akdag M.Z., Dasdag S., Cakir D.U., Kizil M.: Extremely low frequency magnetic fields cause oxidative DNA damage in rats. Int. J. Radiat. Biol., 2008; 84: 789-795

[3] Lin Q., Dong L., Xu Y., Di G.: Studies on effects of static electric field exposure on liver in mice. Sci. Rep., 2018; 8: 15507

[4] Reiter R.J., Abreu-Gonzalez P., Marik P.E., Dominguez-Rodriguez A.: Therapeutic algorithm for use of melatonin in patients with $\mathrm{CO}$ VID-19. Front. Med., 2020; 7: 226

[5] Zisapel N.: New perspectives on the role of melatonin in human sleep, circadian rhythms and their regulation. Br. J. Pharmacol. 2018; 175: 3190-3199

[6] Sun H., Gusdon A.M., Qu S.: Effects of melatonin on cardiovascular diseases: Progress in the past year. Curr. Opin. Lipidol., 2016; 27: 408-413

[7] Sonehara N.M., Lacerda J.Z., Jardim-Perassi B.V., de Paula R.Jr., Moschetta-Pinheiro M.G., Souza Y.S., de Andrade J.C., De Campos Zuccari D.A.: Melatonin regulates tumor aggressiveness under acidosis condition in breast cancer cell lines. Oncol. Lett., 2019; 17: 1635-1645

[8] Morvaridzadeh M., Sadeghi E., Agah S., Nachvak S.M., Fazelian S., Moradi F., Persad E., Heshmati J.: Effect of melatonin supplementation on oxidative stress parameters: A systematic review and meta-analysis. Pharmacol. Res., 2020; 161: 105210

[9] Acuña Castroviejo D., López L.C., Escames G., López A., García J.A., Reiter R.J.: Melatonin-mitochondria interplay in health and disease. Curr. Top. Med. Chem., 2011; 11: 221-240

[10] Anderson G., Vaillancourt C., Maes M., Reiter R.J.: Breast feeding and melatonin: Implications for improving perinatal health. J Breastfeeding Biol., 2016; 1: 8-20 
[11] Zhu D., Ma Y., Ding S., Jiang H., Fang J.: Effects of melatonin on intestinal microbiota and oxidative stress in colitis mice. Biomed. Res. Int. 2018; 2018: 2607679

[12] Öztürk G., Akbulut K.G., Güney Ş.: Melatonin, aging, and COVID-19: Could melatonin be beneficial for COVID-19 treatment in the elderly? Turk. J. Med. Sci., 2020; 50: 1504-1512

[13] Wang J., Wang X., He Y., Jia L., Yang C.S., Reiter R.J., Zhang J.: Antioxidant and pro-oxidant activities of melatonin in the presence of copper and polyphenols in vitro and in vivo. Cells, 2019; 8: 903

[14] Arushanyan É.B.: Melatonin as a drug: Present-day status and perspetives. Eksp. Klin. Farmakol., 2014; 77: 39-44

[15] Osseni R.A., Rat P., Bogdan A., Warnet J.M., Touitou Y.: Evidence of prooxidant and antioxidant action of melatonin on human liver cell line HepG2. Life Sci., 2000; 68: 387-399

[16] TCO Development. TCO Certified Displays 7.0, 2015. http:// tcocertified.com/files/2015/11/TCO-Certified-Displays-7.0.pdf (01.10.2018)

[17] Placer Z., Cushman L.L., Johnson B.C.: Estimation of products of lipid peroxidation (malondialdehyde) in biochemical systems. Anal. Biochem., 1966; 16: 359-364

[18] Misra H.P., Fridovich I.: The role of superoxide anion in the autooxidation of epinephrine and a simple assai superoxide dismutase. J. Biol. Chem., 1972; 247: 3170-3175

[19] Oyewopo A.O., Olaniyi S.K., Oyewopo C.I., Jimoh A.T.: Radiophrequency electromagnetic radiation from cell phone causes defective testicular function in male Wistar rats. Andrologia, 2017, 49: e12772

[20] Hosseinabadi M.B., Khanjani N.: The effect of extremely low-frequency electromagnetic fields on the prevalence of musculoskeletal disorders and the role of oxidative stress. Bioelectromagnetics, 2019; 40: 354-360
[21] Bicer M., Baltaci S.B., Patlar S., Mogulkoc R., Baltaci A.K.: Melatonin has a protective effect against lipid peroxidation in the bone tissue of diabetic rats subjected to acute swimming exercise. Horm. Mol. Biol. Clin. Investig., 2018; 34: 20170079

[22] Sokolovic D., Djordjevic B., Kocic G., Stoimenov T.J., Stanojkovic Z., Sokolovic D.M., Veljkovic A., Ristic G., Despotovic M., Milisavljevic D., et al.: The effects of melatonin on oxidative stress parameters and DNA fragmentation in testicular tissue of rats exposed to microwave radiation. Adv. Clin. Exp. Med., 2015; 24: 429-436

[23] Girish K.S., Paul M., Thushara R.M., Hemshekar M., Shanmuga Sundaram M., Rangappa K.S., Kemparaju K.: Melatonin elevates apoptosis in human platelets via ROS mediated mitochondrial damage. Biochem. Biophys. Res. Commun., 2013; 438: 198-204

[24] Poljsak B., Milisav I.: The neglected significance of 'Antioxidative Stress'. Oxid. Med. Cell. Longev., 2012; 2012: 480895

[25] Ruano-Ravina A., Figueiras A., Freire-Garabal M., Barros-Dios J.M.: Antioxidant vitamins and risk of lung cancer. Curr. Pharm. Des., 2006; 12: 599-613

[26] Kocyigit A., Guler E.M., Karatas E., Caglar H., Bulut H.: Dose-dependent proliferative and cytotoxic effects of melatonin on human epidermoid carcinoma and normal skin fibroblast cells. Mutat. Res. Genet. Toxicol. Environ. Mutagen., 2018; 829-830: 50-60

[27] Bejarano I., Espino J., Barriga C., Reiter R.J., Pariente J.A., Rodríguez A.B.: Pro-oxidant effect of melatonin in tumour leucocytes: Relation with its cytotoxic and pro-apoptotic effects. Basic Clin. Pharmacol. Toxicol., 2011; 108: 14-20

[28] Melatonin capsules. EmpoweR Pharmacy FDA 503B Registered, PCAB Accredited, Compounding Pharmacy. https://www.empowerpharmacy.com/drugs/melatonin-capsules (08.03.2018)

[29] Hardeland R.: Melatonin in aging and disease - multiple consequences of reduced secretion, options and limits of treatment. Aging Dis., 2012; 3: 194-225 